\title{
Acetato de ulipristal en el diagnóstico diferencial de carcinoma de endometrio
}

\author{
Laura Baquedano Mainar ${ }^{1}$, Pilar Del Tiempo Marqués ${ }^{1}$, Ignacio Adiego Calvo ${ }^{1}$, Francisco \\ Villalobos Salguero ${ }^{1}$, Patricia Rubio Cuesta ${ }^{1}$, Esther Gimeno ${ }^{2}$, Miguel Angel Ruiz Conde. ${ }^{1}$ \\ ${ }^{1}$ Hospital Universitario Miguel Servet, Servicio de Ginecología. ${ }^{2}$ Hospital Universitario Miguel Servet, Servicio de \\ Anatomía Patológica. Zaragoza, España.
}

\section{RESUMEN}

Los miomas uterinos son las tumoraciones genitales más frecuentes cuyo tratamiento más habitual es el quirúrgico. Actualmente existe un tratamiento médico eficaz para reducir su volumen y la clínica asociada que es el acetato de ulipristal (AU), que es un modulador selectivo de los receptores de la progesterona. A nivel endometrial puede ocasionar cambios histológicos que cuando son muy marcados plantean dudas diagnósticas. Se presenta el caso de una paciente con útero miomatoso sintomático bajo tratamiento con $\mathrm{AU}$, la histología en las muestras de biopsia planteó el diagnóstico diferencial con adenocarcinoma de endometrio.

\section{SUMMARY}

Uterine fibroids are the most common genital tumors and the most common treatment is surgery. Actually there is an effective medical treatment to reduce its volume and the symptoms. It is ulipristal acetate (UA), a selective progesterone receptor modulator. In the endometrium it can cause some peculiar histological changes. We present a patient with symptomatic uterine fibroid with UA, and it was difficult to make differential diagnosis with endometrial cancer.

\section{INTRODUCCIÓN}

Los miomas uterinos o leiomiomas son los tumores más comunes de la mujer. Son de naturaleza benigna, derivan del músculo liso y tienen dependencia hormonal. Aparecen frecuentemente en la perimenopausia, de tal manera que después de los 40 años una de cada dos mujeres tienen miomas. Cuando son sintomáticos causan sangrado uterino que puede secundariamente provocar anemia, dismenorrea, dolor pélvico y otra sintomatología por compresión como poliaquiuria o tenesmo rectal. Por todo ello los leiomiomas son causa de una merma en la calidad de vida de la mujer y por ello un motivo frecuente de consulta ginecológica $(1,2)$.

El tratamiento más habitual para los miomas sintomáticos sigue siendo hoy en día la cirugía, bien sea conservando el útero o realizando una histerectomía. Teniendo en cuenta su dependencia hormonal en ocasiones se utilizan los análogos de la $\mathrm{GnRH}$ como tratamiento preoperatorio para disminuir su volumen y mejorar la anemia de las pacientes, sin embargo, sus importantes efectos adversos hacen que su utilidad sea limitada.

El acetato de ulipristal (AU) es un modulador 
selectivo de los receptores de la progesterona (MSRP), autorizado recientemente en Europa para el tratamiento de los síntomas moderados o intensos causados por los miomas uterinos en mujeres adultas en edad reproductiva. El tratamiento consiste en la administración de un comprimido de 5 mg vía oral una vez al día durante 3 meses, pudiendo repetirse este ciclo. La eficacia y seguridad del tratamiento ha sido documentada en primera instancia mediante varios ensayos clínicos aleatorizados en los que el AU se compara con placebo y a leuprorelina (análogo $\mathrm{GnRH})(3,4)$. Estos primeros ensayos que constataron la eficacia y seguridad del fármaco valieron para aprobar el fármaco como tratamiento prequirúrgico de los miomas y el estudio más reciente (PEARL IV) ha servido para introducir en ficha técnica la indicación de tratamiento médico intermitente y no sólo de forma preoperatoria (5).

El tratamiento con AU causa cambios específicos de clase en el endometrio conocidos con el nombre de Cambios Endometriales Asociados a los MSRP (CEAP), que revierten al suspender el tratamiento. En algunas pacientes también puede originar un engrosamiento endometrial visible por ecografía que es así mismo reversible. Estos cambios endometriales son a veces de muy difícil valoración sobre todo teniendo en cuenta que se trata de un fármaco recientemente introducido en la práctica clínica diaria y su experiencia a la hora del estudio anatomopatológico es limitada.

Presentamos el caso de una paciente en tratamiento con AU que planteó problemas de diagnóstico diferencial con un adenocarcinoma endometrioide de tipo mucinoso.

\section{Caso clínico}

Paciente de 49 años, que es remitida desde el Hospital Obispo Polanco de Teruel, por diagnóstico anatomopatológico de adenocarcinoma de endometrio mucinoso de tipo endocervical bien diferenciado (70\% mucinoso, 30\% endometrioide) y masa pélvica con el mismo diagnóstico anatomopatológico.

No destacaba ningún antecedente familiar ni personal de interés excepto que era nuligesta, con historia de infertilidad y refería ciclos menstruales normales hasta el último año. Mantenía controles en su centro Médico de Especialidades de referencia por útero miomatoso que permanecía estable en las pruebas de imagen, aunque en los últimos meses provocaba anemia leve por lo que paciente tomaba sulfato ferroso vía oral. Se indicó un ciclo de tratamiento de 3 meses con $5 \mathrm{mg}$ oral de AU con objeto de remontar la anemia de forma preoperatoria y que inició en abril de 2015, con la intención de realizar un tratamiento quirúrgico definitivo 3 meses después. Ante la persistencia del sangrado genital anómalo, se indica tratamiento quirúrgico el 29 de mayo.
A la apertura de la cavidad abdominal se objetiva un síndrome adherencial severo con pelvis congelada que dificulta acceder a la misma. El útero está fijo y aumentado de tamaño e íntimamente adherido a recto y a sigma. Los anexos son de aspecto tumoral y están adheridos a la cara posterior y lateral uterina. Debido a la dificultad técnica quirúrgica y la alta sospecha de malignidad se decide realizar biopsia de las masas ováricas y legrado uterino fraccionado en el mismo acto quirúrgico. El resultado del análisis anatomopatológico de ambas muestras de tejido es de adenocarcinoma mucinoso de tipo endocervical bien diferenciado (G1). Por ello la paciente es remitida al Hospital Universitario Miguel Servet de Zaragoza.

Aporta un TAC abdominopélvico realizado en el Hospital de Teruel en el que se informa de útero polimiomatoso, con miomas entre 2,5 y $4 \mathrm{~cm}$. En fondo y cara lateral izquierda existe masa de $6,5 \mathrm{~cm}$ de diámetro máximo, heterogénea que contacta y comprime sigma y parece depender de útero sin poder descartar origen anexial. Los anexos no se delimitan claramente y parece estar todo el aparato genital englobado en la misma masa pélvica (Figura 1).

Al examen se aprecia una paciente con sobrepeso (IMC 29), que en el momento de la consulta presentaba un sangrado genital importante. Al tacto bimanual se objetiva una tumoración dura, fija, de unos 9-10 cm dolorosa al tacto que engloba todo el aparato genital interno y por ecografía se confirman los hallazgos del TAC. En la colonoscopia no se detecta ninguna lesión endoluminal y se visualiza una compresión extrínseca del sigma con mucosa normal. Los marcadores tumorales están alterados: Ca 12.5 de 73,3 U/ml; Ca 19.9 de $76 \mathrm{U} /$ $\mathrm{ml}$; HEA de 172 pmol/L; Ca 15.3 de 25,2 U/ml. El resto del estudio preoperatorio fue normal.

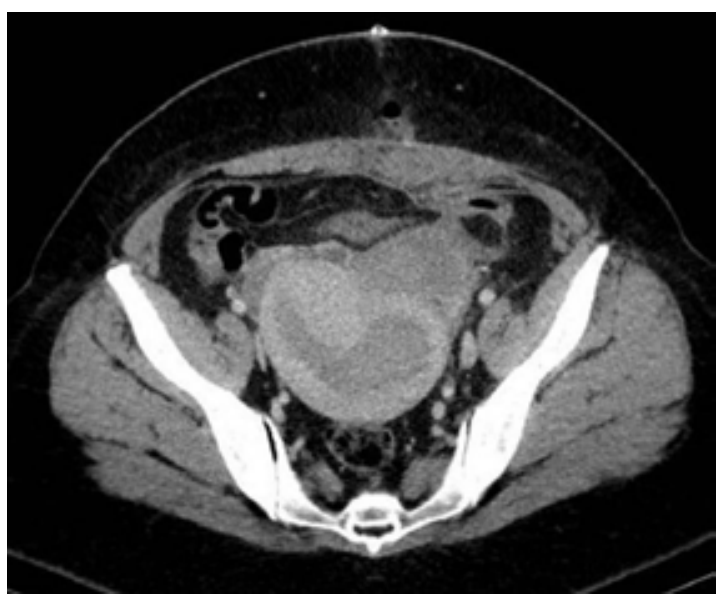

Figura 1. TAC abdominopélvico. Utero miomatoso. Imagen de masa anexial izquierda. 
Es intervenida mediante laparotomía media infraumbical en la que se objetiva el útero aumentado de tamaño, íntimamente adherido a la vejiga, con múltiples miomas subserosos e intramurales y tumoraciones quísticas en ambos ovarios de 4 y $5 \mathrm{~cm}$ con apertura de quiste de ovario derecho y salida de abundante material mucinoso oscuro a cavidad abdominal. Existen adherencias firmes de anexo izquierdo a recto y sigma y de asas intestinales a pared abdominal, por lo que se requiere la colaboración del Servicio de Cirugía General. Se realiza adhesiolisis e histerectomía total simple y doble anexectomía posterior sin dejar tumor residual.

En la descripción macroscópica de la pieza quirúrgica se objetiva varios nódulos en cuerpo uterino de entre 1 y $4 \mathrm{~cm}$ que corresponden a leiomiomas; en los anexos existe una fibrosis importante de mesosalpinx y mesoovario y quistes en ambos ovarios, el mayor de $6 \mathrm{~cm}$ en ovario izquierdo con contenido mucoso-hemorrágico de coloración negruzca. El análisis histológico plantea serias dudas diagnóstico diferencial con la hiperplasia endometrial y el adenocarcinoma de endometrio debido a la presencia de pequeñas papilas vellosas en la muestra. Tras un estudio exhaustivo por varios patológos y la consulta a la Unidad de Anatomía Patológica de otro centro Hospitalario, se emite un diagnóstico final de hiperplasia endometrial compleja con y sin atipia (neoplasia endometrial intraepitelial), con importante metaplasia mucinosa, papilar y eosinofílica. Además existen leiomiomas intramurales con adenomiosis superficial y profunda y ambas trompas con implantes endometriósicos y quistes ováricos endometriósicos, todos ellos con importante metaplasia mucinosa, papilar y eosinofílica muy similar a los cambios mostrados en el endometrio (Figura 2 y 3 ).

En el postoperatorio la paciente presenta anemia que es tratada con hierro endovenoso y oral,

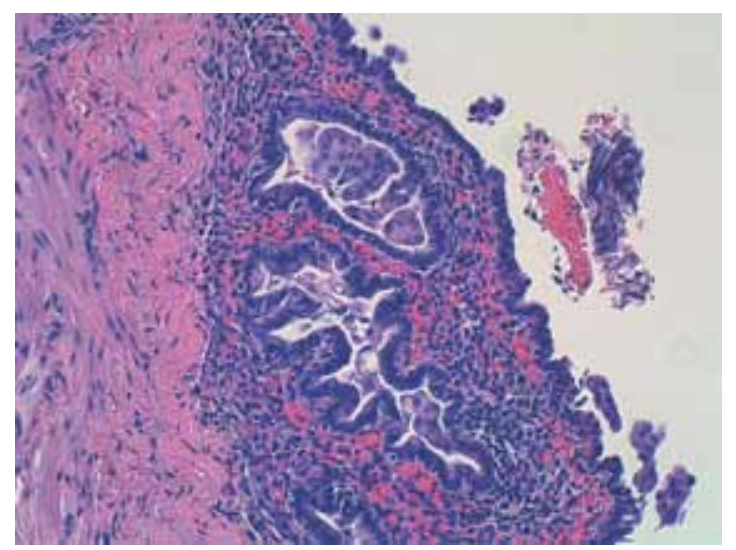

Figura 2. Anexo con la lesión hiperplásica, metaplásica y endometriosis. HE 200x.

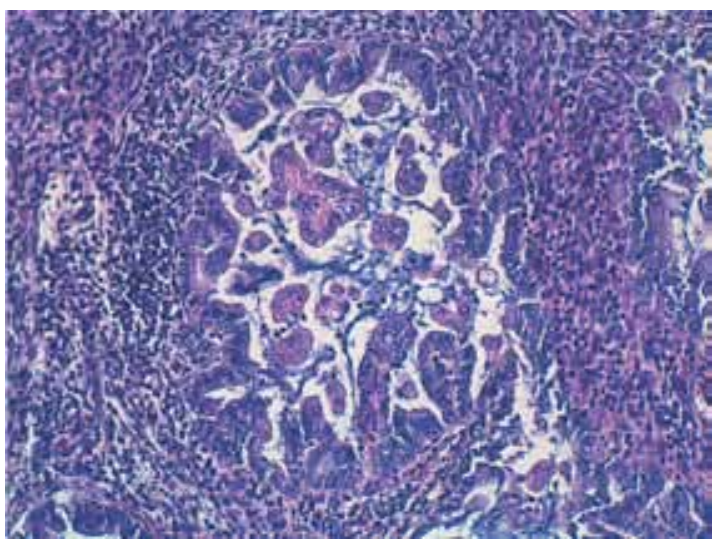

Figura 3. Endometrio con hiperplasia compleja. HE 200x.

e infección de la herida quirúrgica, la que una vez resuelta con antibiótico terapia es dada de alta a su domicilio.

\section{DISCUSIÓN}

El AU es un MSRP y como tal tiene actividad agonista o antagonista de la progesterona en función del tejido diana. Los miomas uterinos tienen receptores de progesterona y el $\mathrm{AU}$ se une a ellos provocando un efecto antagonista de la progesterona favoreciendo un efecto antiproliferativo y apoptótico que se traduce en una reducción del tamaño de los mismos. Además impide los picos de LH y la ovulación induciendo amenorrea (3).

El endometrio es un tejido diana importante de la acción de los MSRP en el que ejerce efectos específicos que pueden incluir engrosamiento endometrial, con traducción ecográfica en ocasiones, y de características histológicas similares a la hiperplasia quística (6). Estos cambios específicos de clase se han denominado Cambios Endometriales Asociados a los MSRP o CEAP (PAEC en la terminología inglesa). Estos cambios endometriales tan representativos pueden aparecer hasta en un $60 \%$ de las pacientes y son reversibles tras la suspensión del tratamiento (6-10).

Los CEAP se caracterizan por: A. Glándulas endometriales con arquitectura irregular, frecuentemente con extensa dilatación quística, mayoritariamente difusas. B. Epitelio glandular con aspecto inactivo, con células epiteliales cuboides bajas, no estratificadas con pocas mitosis. Este es el rasgo principal de los CEAP en el que se suele basar el diagnóstico diferencial con la hiperplasia endometrial y el endometrio estimulado por estrógenos sin oposición con gestágenos. En algunas ocasiones puede observarse metaplasia ciliada que afecta particularmente al recubrimiento epitelial de las 
glándulas con dilatación quística. C. Aspecto secretor con glándulas en espiral o tortuosas, semejantes a la fase secretora, con vacuolización citoplasmática de las células glandulares. La luz de las glándulas quísticas puede contener secreción acuosa. D. Diseminación irregular de las glándulas por todo el estroma con celularidad densa y sin cambio predecidual.

Los estudios de eficacia (PEARL I-IV) muestran que $A U$ es un tratamiento seguro a nivel endometrial aunque provoca los cambios descritos que es necesario conocer $(3-5,10)$. Durante dichos estudios, se realizaron por protocolo biopsias de endometrio de control antes y después de los ciclos trimestrales de tratamiento que fueron analizadas por 3 patólogos a ciegas y de forma independiente. Fueron informadas características no propias del endometrio fisiológico en porcentajes variables (11-25\%) en las biopsias de control realizadas al finalizar los ciclos con AU. En sólo una paciente un patólogo informó de posible adenocarcinoma de endometrio después del tratamiento con AU. Sin embargo el mismo patólogo emitió el mismo informe en la biopsia inicial, que después se descartó, por lo que no se trata de un efecto derivado del tratamiento (5).

No existen hasta la fecha estudios con AU y endometrioisis, pero dado que se trata de la aparición de tejido endometrial extrauterino es de prever que los mismos cambios histológicos que puede ocasionar el $\mathrm{AU}$ en el endometrio se verán reflejados en aquellas localizaciones donde se encuentre este tejido activo.

\section{CONCLUSIONES}

EI $A U$ induce cambios en el endometrio que es necesario conocer. Si el patólogo no está familiarizado con dichos cambios o desconoce que la paciente ha llevado el tratamiento con AU, puede confundir la histología con la de una hiperplasia con o sin atipia o incluso un carcinoma de endometrio. Por ello resulta fundamental la buena comunicación entre el ginecólogo y el patólogo para entender los hallazgos microscópicos de la muestra de tejido que se remite, bien sea una biopsia o la pieza definitiva.

\section{REFERENCIAS}

1. Downes E, Sikirica V, Gilabert- Estelles J, Bolge SC, Dodd $\mathrm{SI}$, Mroulis $\mathrm{C}$, et al. The burden of uterine fibroids in five European countries. Eur J Obstet Gynecol Reprod Biol 2010;152:96-102.

2. Spies JB, Coyne K, Guaou GN, Boyle D, SkyrnarzMurphy K, Gonzalves SM, et al. The UFS-QOL, a new disease-specific symptom and health-related quality of life questionnaire for leiomyomata. Obstet Gynecol 2002;99:290-300.

3. Donnez J, Tatarchuk TF, Bouchard P, Puscasiu L, Zakharenko NF, Ivanova T, et al. Ulipristal acetate versus placebo for fibroid treatment before surgery. $\mathrm{N}$ Engl J Med 2012;366:409-20.

4. Donnez J, Tomaszewski J, Vazquez F, Bouchard P, Lemiezczuk B, Baro F, et al. Ulipristal acetate versus leuprolide acetate for uterine fibroids. $\mathrm{N}$ Engl $\mathrm{J}$ Med 2012;366:421-32.

5. Donnez J, Hudecek R, Donnez O, Matule D, Arhendt HJ, Zatik J. Efficacy and safety of repeated use of ulipristal acetate in uterine fibroids. Fertil Steril 2015;103:519-27.

6. Mutter GL, Bergeron C, Deligdisch L, Ferenczy A, Glant M, Merino M, et al. The spectrum of endometrial pathology induced by progesterone receptor modulators. Mod Pathol 2008;21:591-8.

7. Mutter GL, Bergeron C, Deligdisch L, Ferenczy A, Glant M, Merino M, et al. The spectrum of endometrial pathology induced by progesterone receptor modulators. Mod Pathol 2008;21:591-8.

8. Fiscella J, Bonfiglio T, Winters P, Eisinger SH, FisceIla K. Distinguishing features of endometrial pathology after exposure to the progesterone receptor modulator mifepristone. Hum Pathol 2011;42:947-53.

9. Williams AR, Bergeron C, Barlow DH, Ferenczy A. Endometrial morphology after treatment of uterine fibroids with the selective progesterone receptor modulator, ulipristal acetate. Int J Gynecol Pathol 2012;31:556-69.

10. Donnez J, Vazquez F, Tomaszewski J, Nouri K, Bouchard P, Fauser BC, et al. Long-term treatment of uterine fibroids with ulipristal acetate. Fertil Steril 2014;101:1565-73. 